Case Report

\title{
Unusual Neurological Manifestation of Acute Respiratory Syndrome Coronavirus 2 (SARS- CoV-2) Infection in a Postpartum Woman: A Case Report
}

\author{
Shalini Shakarwal', Shakun Tyagi ${ }^{2}$, Poonam Sachdeva ${ }^{3}$, Sakshi Aggarwal $^{4}$
}

${ }^{1}$ Assistant Professor, ${ }^{2}$ Associate Professor, ${ }^{3}$ Senior Consultant, ${ }^{4}$ Post Graduate Resident, Department of Obstetrics and Gynaecology, MAMC and Associated Lok Nayak Hospital, New Delhi, India.

DOI: https://doi.org/10.24321/2349.7181.202103

\section{I $\quad \mathbf{N} \quad \mathbf{F} \quad \mathbf{O}$}

Corresponding Author:

Shalini Shakarwal, Department of Obstetrics and Gynaecology, MAMC and Associated Lok Nayak Hospital, New Delhi, India.

E-mail Id:

shakarwal2001@gmail.com

Orcid Id:

https://orcid.org/0000-0002-2648-0183

How to cite this article:

Shakarwal S, Tyagi S, Sachdeva P, Aggarwal S. Unusual Neurological Manifestation of Acute Respiratory Syndrome Coronavirus 2 (SARSCoV-2) Infection in a Postpartum Woman: A Case Report. J Adv Res Med. 2021;8(1):19-21.

\section{$\begin{array}{llllllll}\mathbf{A} & \mathbf{B} & \mathbf{S} & \mathbf{T} & \mathbf{R} & \mathbf{A} & \mathbf{C} & \mathbf{T}\end{array}$}

Severe acute respiratory syndrome coronavirus 2 (SARS-CoV-2) is responsible for the global spread of the disease (COVID-19) at present. Our understanding of the impact of the virus on the nervous system is limited. The involvement of CNS and neurological manifestations reports are further limited in obstetrical cases. We are reporting a case of an unusual presentation of COVID-19 infection in an admitted P2L2 postpartum woman who developed multiple episodes of seizures on postpartum day 8 of lower segment caesarean section, with no prior history of seizures or hypertension. This report will add to a rare neurological manifestation of COVID-19 in pregnancy.

Keywords: CovID-19, Coronavirus, Neurological

Date of Submission: 2021-02-31

Date of Acceptance: 2021-03-28

\section{Introduction}

Severe acute respiratory syndrome coronavirus 2 (SARSCoV-2) is a viral infection with rapid spread and high infectivity. The first case was detected in China (Wuhan) and advanced into a global pandemic. Concerning COVID-19, early available data did not indicate that pregnant individuals were at an increased risk of infection or severe morbidity as compared to non-pregnant individuals in the general population. However, a recent data analysis from the Centers for Disease Control and Prevention (CDC) COVID-19 surveillance suggests that among women with
COVID-19, pregnant women appear to be at an increased risk for certain manifestations of severe illness including CNS involvement as compared to the non-pregnant women. Based on the spectrum of symptoms, in pregnancy, the illness severity included mild, severe, and critical disease. Mild disease was characterised by minimal symptoms and stable vital signs, severe disease was characterised by significant dyspnoea and hypoxia, whereas critical disease was characterised by respiratory failure, shock, or multiorgan system dysfunction.

The Society for Maternal and Fetal Medicine (SMFM) 
has additionally put forth a similar severity scale for the assessment of pregnant patients with COVID-19 including 5 categories, asymptomatic, mild, moderate, severe, and critical. ${ }^{1}$

\section{Mechanism of Nervous System Invasion}

Based on the data available,2 SARS virus, once in blood stream, can spread into central and peripheral nervous systems through retrograde axonal transport or through blood-brain barrier (BBB). Neuro-invasion of SARSCoV-2 may also be via the olfactory nerve or infection of vascular endothelium. The most studied pathway for infection involves binding to the receptor angiotensinconverting enzyme 2 (ACE2), which together with other components of angiotensin system is expressed in the CNS, mostly by endothelial cells but also by both neurons and neuroglial cells. Besides direct invasion of neural cells, SARS-CoV-2 virus affects the neurovascular unit and CNS through systemic inflammation. The major feature of the systemic infection in COVID-19 is the massive increase of pro-inflammatory factors in the circulating blood, often described as a "cytokine storm".

\section{Neurological Manifestation}

As per available data, neurologic symptoms may present as central nervous system manifestations (dizziness, headache, impaired consciousness, acute cerebrovascular disease, ataxia, and seizure), peripheral nervous system manifestations (taste impairment, smell impairment, vision impairment, and nerve pain), and skeletal muscle injury. If we see the overall symptomatology in pregnancy, most of the COVID-19 patients show mild to moderate respiratory symptoms, while very less progress to severe to fatal respiratory and systemic illnesses with no data available on neurological manifestations. The severity of illness depends on the extent or phase of infection, the delay in reporting to the medical health facility and inadequate management in low setup areas. Various strains of coronaviruses have neurotropism and neuroinvasive characteristics resulting in neurological and psychological consequences in a subset of COVID-19 affected population.

\section{Case Presentation}

A 24 year old, P2L2 patient was referred from a government hospital on post-operative day 3 of elective LSCS, done for the indication of previous LSCS and not willing for VBAC. The patient tested RTPCR positive for COVID-19 on postoperative day 3 , for which she was referred to our institution for further management. On admission, she complained of cough since 3 days and had a single episode of fever 2 days back. Her present pregnancy was uneventful, with no history of high BP, seizures, or any other medical and/ or surgical illness and no significant past or family history.
On examination, she was conscious, oriented, with stable vitals and no pallor, oedema, and icterus. Per abdominal examination was unremarkable and no other abnormalities were detected. She maintained oxygen saturation on room air. She was kept under observation and her all laboratory parameters were within normal limits. She was started on treatment as per the institutional protocol along with vitals and oxygen saturation monitoring. On day 4 of admission, she developed 3-4 episodes of generalised tonic-clonic seizures. Seizures were preceded by a single episode of vomiting. At the time of seizures, her BP shot up once to $142 / 114$, which was controlled with a single dose of injectable labetalol.

She was started on injection dexamethasone and injection LMWH according to treatment for the moderate category of COVID-19 along with intravenous phenytoin. Seizures were controlled. Once stabilised, X-ray chest PA view and NCCT head was done in emergency and no abnormality was detected. Repeat blood investigations also came within normal limits. BP charting was done and the patient remained normal thereafter, without being on any antihypertensive drugs. Contrast CT scan of the head was done the day after the episode and no evidence of any concealed intraventricular haemorrhage was found.

Figures 1 (a) and (b) show the contrast-enhanced CT images of the patient, which show normal findings.

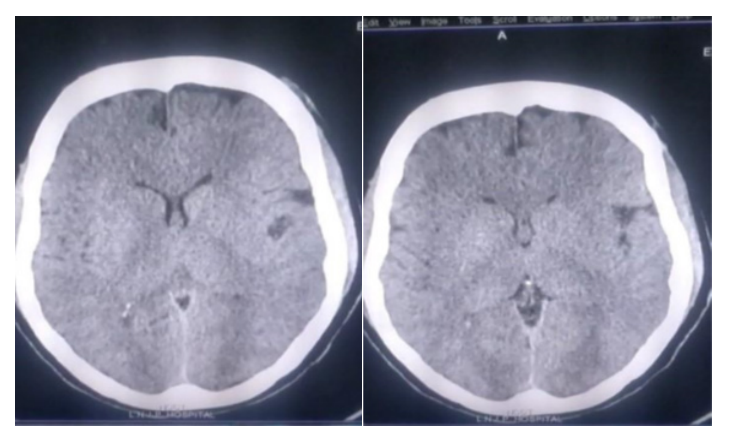

(a)

(b)

\section{Figures I.(a) and (b) Contrast-enhanced Computer Tomography Images}

The patient was monitored for the next few days and a repeat RTPCR test was done on post-operative day 10 which was negative. She was shifted in a satisfactory condition to a non-COVID-19 hospital with a neurology department after discussing with the department head. She was on oral phenytoin, antibiotics, and supplements at the time of referral.

\section{Discussion}

Though the common presentation of COVID-19 is respiratory complaints and influenza-like symptoms, now, after many 
cases, other symptoms like anosmia or ageusia should also raise suspicion of a COVID infection. In a first report3 from Wuhan (China), out of 214 COVID-19 infected patients, it was reported that the incidence of neurological damage caused by SARS-CoV-2 was $36.4 \%$ and fell into 3 categories: central nervous system (dizziness, headache, impaired consciousness, acute cerebrovascular disease, ataxia, and seizure), peripheral nervous system (taste impairment, smell impairment, vision impairment, and nerve pain), and musculoskeletal. Uncommonly, COVID-19 can also present with central nervous system manifestations such as ischemic stroke, intracerebral haemorrhage, encephalomyelitis, and acute myelitis, peripheral nervous manifestations such as Guillain-Barré syndrome and Bell's palsy, and skeletal muscle manifestations such as rhabdomyolysis. Specifically, more severe patients were likely to have neurologic symptoms such as cerebrovascular diseases, consciousness impairment, and skeletal muscle symptoms. Out of two systemic reviews of neurological manifestations in COVID-19 infection, in one where a total of 841 patients with neurological manifestation in COVID-19 were studied, the most commonly reported were headache, ischemic strokes, disorders of consciousness, peripheral nervous system manifestations and rare cases of seizures $(0.7 \%)$, in which most of them were having severe COVID-19 infection. ${ }^{4}$ In another systemic review, ${ }^{5}$ that included data from 37 articles, the most commonly reported neurological manifestations of COVID-19 were myalgia, headache, altered sensorium, hyposmia, hypogeusia, and uncommonly central nervous system manifestations such as ischemic stroke, intracerebral haemorrhage, encephalomyelitis, acute myelitis, Guillain-Barré syndrome, Bell's palsy, and rhabdomyolysis. In this review ${ }^{5}$ also, there was one case of epilepsy $(0.5 \%)$ which is reported in a single study3. As far as our knowledge is concerned, no data is available on neurological manifestations in pregnant females. There is only one case report of seizures in pregnancy due to COVID-19, ${ }^{6}$ but that too in antenatal period. We are presenting the only postpartum case with COVID-19 infection complicated with tonic-clonic seizures.

After seeing all the data available to us, our case is unique in presentation. Since our patient had no history of seizure and was normotensive, it does fall in the category of epilepsy or pre-eclampsia - eclampsia spectrum. In most of the cases of neurological manifestations, the patient usually suffers from severe illness and other co-morbidities but in our case, no other co-morbidities were there apart from the postpartum state.

\section{Conclusion}

Although it is a single case report, it can be taken as a rare neurological manifestation of COVID-19 infection in postpartum case. We need to maintain close neurologic surveillance for prompt recognition of these complications. The mechanisms and consequences of COVID-19 infection causing neurologic involvement require further studies.

\section{Conflict of Interest: None \\ References}

1. ACOG [Internet]. Novel Coronavirus 2019 (COVID-19). Available from: www.acog.org/clinical/clinicalguidance/practice-advisory/articles/2020/03/novelcoronavirus-2019

2. Tremblay ME, Madore C, Bordeleau M, Tian L, Verkhratsky A. Neuropathobiology of COVID-19: The Role for Glia. Front Cell Neurosci. 2020 Nov;14:592214. [PubMed] [Google Scholar]

3. Mao L, Jin $H$, Wang $M, H u Y$, Chen $S$, He Q, Chang J, Hong C, Zhou Y, Wang D, Miao X, Li Y, Hu B. Neurologic Manifestations of Hospitalized Patients with Coronavirus Disease 2019 in Wuhan, China. JAMA Neurol. 2020;77(6):683-90. [PubMed] [Google Scholar]

4. Nepal G, Rehrig JH, Shrestha GS, Shing YK, Yadav JK, Ojha R, Pokhrel G, Tu ZL, Huang DY. Neurological manifestations of COVID-19: a systematic review. Crit Care. 2020;24(1):421. [PubMed] [Google Scholar]

5. Romero-Sanchez CM, Diaz-Maroto I, Fernandez-Diaz E, Sanchez-Larsen A, Layos-Romero A, Garcia-Garcia J, Gonzalez E, Redondo-Penas I, Perona-Moratalla $A B$, Valle-Perez JAD, Gracia-Gil J, Rojas-Bartolome L, Feria-Vilar I, Monteagudo M, Palao M, Palazon-Garcia E, Alcahut-Rodriguez C, Sopelana-Garay D, Moreno Y, Ahmad J, Segura T. Neurologic manifestations in hospitalized patients with COVID-19: The ALBACOVID registry. Neurology. 2020 Aug;95(8):e1060-70. [PubMed] [Google Scholar]

6. Rodriguez AG, Contreras SM, Manovel SMF, Vidal JMM, Buron FD, Fernandez CF, Gonzalez MDCR. SARS-COV-2 infection during pregnancy, a risk factor for eclampsia or neurological manifestations of COVID-19? Case report. BMC Pregnancy Childbirth. 2020 Dec;20(1):587. [PubMed] [Google Scholar] 